\title{
DAP-kinase: from functional gene cloning to establishment of its role in apoptosis and cancer
}

\author{
0 Cohen ${ }^{1}$ and A Kimchi ${ }^{*, 1}$ \\ ${ }^{1}$ Department of Molecular Genetics, Weizmann Institute of Science, Rehovot \\ 76100 , Israel \\ * Corresponding author: A Kimchi, Weizmann Institute of Science, Rehobot \\ 76100, Israel. Tel: 972-8-9342428; Fax: 972-8-9344108; \\ E-mail: Ivkimchi@weizmann.weizmann.ac.il
}

Received 5.5.00; revised 1.8.00; accepted 7.8.00

Edited by G Melino

\begin{abstract}
DAP-kinase is a pro-apoptotic $\mathrm{Ca}^{2+}$ calmodulin-regulated serine/threonine kinase that participates in a wide array of apoptotic systems initiated by interferon- $\gamma$, TNF- $\alpha$, activated Fas, and detachment from extracellular matrix. It was isolated by an unbiased functional approach to gene cloning aimed at hitting central mediators of the apoptotic process. This $160 \mathrm{Kd}$ protein kinase is localized to actin microfilaments and carries interesting modules such as ankyrin repeats and the death domain. The death promoting effects of DAP-kinase depend on its intact catalytic activity, the correct intracellular localization, and on the presence of the death domain. A few mechanisms restrain the killing effects of the protein in healthy cells. The enzyme's active site is negatively controlled by an adjacent $\mathrm{CaM}$ regulatory domain whose effect is relieved by binding to $\mathrm{Ca}^{2+}$-activated calmodulin. A second mode of autoinhibition engages the serine-rich C-terminal tail, spanning the last 17 amino acids of the protein. A link between DAPkinase and cancer has been established. It was found that the mRNA and protein expression is frequently lost in various human cancer cell lines. Analysis of the methylation status of DAP-kinase's $5^{\prime}$ UTR in DNA extracted from fresh tumor samples, showed high incidence of hypermethylation in several human carcinomas and $B$ cell malignancies. The antitumorigenic effect of DAP-kinase was also studied experimentally in mouse model systems where the re-introduction of DAP-kinase into highly metastatic mouse lung carcinoma cells who had lost the protein, strongly reduced their metastatic capacity. Thus, it appears that loss of DAP-kinase confers a selective advantage to cancer cells and may play a causative role in tumor progression. A few novel kinases sharing high homology in their catalytic domains with DAPkinase have been recently identified constituting altogether a novel family of death promoting serine/threonine kinases. Cell Death and Differentiation (2001) 8, 6-15.
\end{abstract}

Keywords: apoptosis; DAP-kinase; cancer; microfilaments; metastasis; functional gene cloning

Abbreviations: DAP, Death Associated Protein; CaM, calmodulin; IFN, interferon; TKO, technical knock out; TNF, tumor necrosis factor; DRP-1, DAP-kinase related protein-1

\section{The technical knock out strategy}

The final cell number in multicellular organisms is determined by the controlled balance between loss and gain of cells. Therefore, cell death resulting from the active process of apoptosis, along with growth, proliferation and differentiation processes, is a fundamental aspect in the life cycle of eukaryotic cells. The molecular mechanisms leading to the controlled removal of cells by apoptosis in embryonic and adult tissues was hardly understood several years ago. Yet, in the past few years enormous progress has been made in identifying the individual molecular components of the apoptotic pathways. Classical genetic tools, applied in $C$. elegans and Drosophilla (reviewed $\mathrm{in}^{1}$ ), were most powerful in deciphering some of these components, the isolation of which was followed in most cases by the identification of their mammalian orthologs. In parallel, biochemical assays in cell free systems, and systematic screens for novel proteins which directly interact with already known proteins, provided other independent approaches which further extended the molecular information and highlighted its complexity in higher organisms. $^{2,3}$ In addition, we have witnessed the development of novel approaches of functional gene cloning which were applied in cell cultures undergoing apoptosis in response to a defined stimulus. ${ }^{4}$ The latter approaches were aimed at harnessing the strong power which resides in genetic screens to the rescue of apoptotic genes from mammalian cell systems. To overcome the lack of the classical genetic tools which turned out to be very powerful in invertebrates, novel strategies of random gene inactivation were further designed instead of mutating the DNA itself (reviewed $\mathrm{in}^{4}$ ). They were aimed at targeting the mRNA transcripts by introducing into cells anti-sense cDNA libraries or at inactivating the protein function by short sense cDNA fragments. The first functional approach to gene cloning developed and utilized for the rescue of apoptotic genes was named Technical Knock Out (TKO) strategy. ${ }^{5}$ It led to the identification of a few novel apoptotic genes to one of which this review is devoted.

The TKO genetic screen method is based on random gene inactivation by transfections with expression libraries comprising anti-sense cDNA fragments. The assumption is that specific anti-sense RNA mediated inactivation of a rate limiting death-promoting gene would confer some growth 
advantage to cells that are exposed to an apoptotic stimulus. This advantage can then be used as a powerful forward selection to rescue the relevant death-protective anti-sense cDNA and subsequently to identify the corresponding protein whose continued expression is necessary for apoptosis. Using the TKO approach in a model system of human HeLa cervical carcinoma cells and IFN $-\gamma$ as the killing agent, several novel Death Associated Proteins (DAP's) were identified. These genes share one common property which served as the basis for their selection, i.e., their inactivation had weakened the apoptotic phenotype suggesting that each of them controls a rate limiting process in the apoptotic network. Yet, a detailed study performed on these genes over the past few years revealed that these genes display a wide spectrum of cellular and biochemical functions, as summarized in some recent reviews. ${ }^{4,6}$ For example, DAP-5 is an homologue of the elF4GI translation initiation factor and is responsible for critical changes in protein translation which occur during apoptosis. It drives IRES-dependent translation under conditions where cap-dependent translation is turned off. $^{7,8}$ DAP-3 is a novel nucleotide-binding protein whose intact $P$-loop motif is necessary for its death-promoting function. ${ }^{9,10}$ Another gene that was isolated by this screen as a positive mediator of cell death is cathepsin $D-$ an aspartic lysosomal protease which accumulates at high levels in an unprocessed active form during apoptosis. ${ }^{11}$ This review will focus on yet another novel gene isolated by this screen, named DAP-kinase. We will describe the multidomain structure and the biochemical properties of this protein, discuss its involvement in apoptosis, and relate the implication of its loss or inactivation to cancer development. Moreover, the recent discovery of several members which belong to the growing DAP-kinase family of proteins will be described, focusing on the apoptotic functions of these serine/threonine kinases.

\section{The multi-domain structure of DAP-kinase}

One of the anti-sense cDNA fragments which conferred partial protection from IFN- $\gamma$-induced cell death served to clone a novel gene that was named DAP-kinase. ${ }^{12}$ Once the full length cDNA clone of DAP-kinase was isolated, the deduced amino acid structure predicted that a novel calcium/ calmodulin-dependent serine/threonine type kinase (160 kDa) carrying an interesting combination of structural motifs had been isolated (see Figure 1A). Its kinase domain has a classical 12 subdomain composition, typical of serine/ threonine kinases, followed by a region that shares high homology with the calmodulin regulatory domains of other kinases. Adjacent to the latter, eight ankyrin repeats were identified, followed by two P-loop motifs. ${ }^{12}$ At the carboxy terminus of the protein, a death domain was identified, containing all the boxes of homology and the conserved amino acids characteristic of analogous domains in other proteins. $^{13}$

The ankyrin repeats, identified so far in a long list of different intracellular and extracellular proteins, create a distinct module mediating protein-protein interactions. The molecular basis of these interactions was recently studied
A.

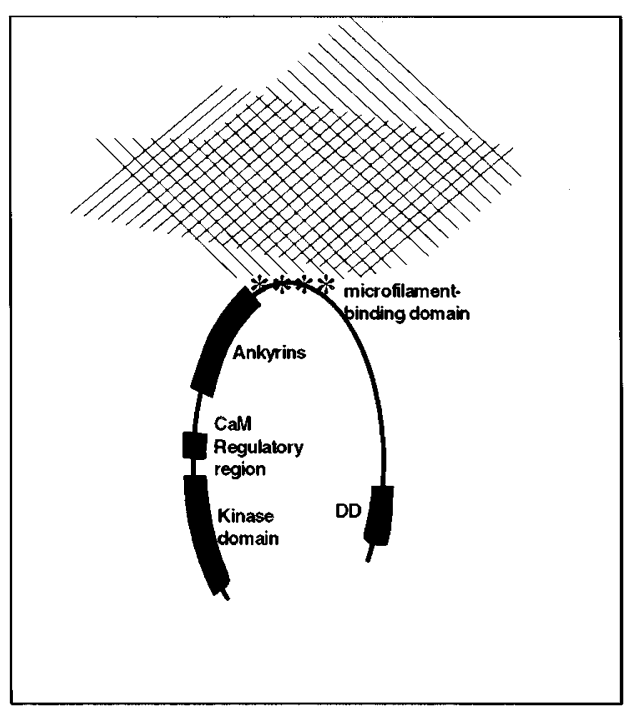

B.

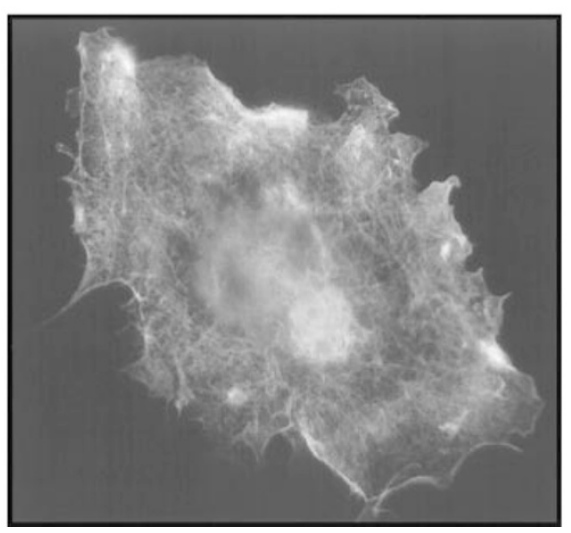

Figure 1 (A) Schematic representation of DAP-kinase protein. The various motifs and domains as predicted by the deduced amino acid sequence and/or experimental work are shown. The crossed lines represents the actinmicrofilament network. (B) Intracellular localization of DAP-kinase. COS cells were transfected with FLAG tagged DAP-kinase and immunostained with antiFLAG antibodies $48 \mathrm{~h}$ later

in detail based on the X-ray crystallography of ankyrin repeats from various proteins. ${ }^{14}$ In DAP-kinase, this motif may mediate interactions with downstream or upstream specific effectors. The two P-loop motifs suggest that the protein may possess additional nucleotide-binding regions outside of the catalytic domain. The death-domain module, identified at the $3^{\prime}$ end of DAP-kinase represents another motif that mediates protein-protein interactions by either homo- or heterodimerizations. Many of these proteins have a direct involvement in programmed cell death including the p55 TNF receptor, the Fas/APO-1 receptor, DR3-5, FADD/ MORT-1, RIP and TRADD. ${ }^{15}$ Since no indications for homodimerization were so far detected as detailed below, 
the death domain of DAP-kinase may prompt association with other protein partners critical for the function of this novel type of kinase. Interestingly, a stretch of amino acids at the carboxy end of the protein which is rich in serines follows the death domain, a feature characteristic of many of the C-terminal tails in various death domain-containing proteins. ${ }^{13}$ Recently we found that deletion of the last 17 amino acids from the full length protein generated a gain of function mutation which promoted very significantly the apoptotic effects of DAP-kinase without affecting its catalytic activity. ${ }^{16}$ The serine rich C-terminal tail of DAPkinase therefore seems to function as an autoinhibitory module which negatively regulates a putative function of the protein residing in one of the various extra-catalytic domains. The death domain could be a potential target for auto-inhibition by the C-terminal tail, as previously shown for the corresponding region in the Fas/APO-1 receptor. ${ }^{17}$

The overall structure of human DAP-kinase is unique and has no known analogy to other proteins. The mouse and the $C$. elegans DAP-kinase orthologs were recently identified and cloned in our laboratory ( $B$ Inbal and $A$ Kimchi 2000, unpublished results). The protein products of these genes show remarkable homology to the human protein (approximately 92 and 34\% identity at the amino acid level for the mouse and $C$. elegans proteins, respectively). This homology is not confined to a restricted region, but rather spreads all over the protein, preserving all the functional domains, indicating an evolutionary conservation of the protein.

The complex structure and the high conservation in evolution, emphasized the importance of studying this novel death-associated protein both at the biochemical and functional level.

\section{The catalytic activity is essential for the death-promoting function of DAP-kinase}

The biochemical properties of the catalytic domain of DAPkinase were assessed in cell free systems. In the first stage, in vitro kinase assays with a recombinant wild-type protein were performed. These assays proved that DAP-kinase undergoes autophosphorylation and is capable of phosphorylating a chosen exogenous substrate - the myosin light chain (MLC). The autophosphorylation of the full length protein as well as the phosphorylation of MLC were both stimulated by $\mathrm{Ca}^{2+}$ / calmodulin. ${ }^{18}$ Specific calmodulin overlay assays subsequently proved that the recombinant protein was capable of directly binding radiolabeled calmodulin. ${ }^{18}$ The removal of the calmodulin regulatory domain ( $\triangle \mathrm{CaM}$ mutant) generated a constitutively active kinase when assessed in the MLC phosphorylation assays (i.e., a gain of function mutation). This is consistent with previous information on other well studied calmodulin-dependent kinases, in which the calmodulin-regulatory domain displays inhibitory effects on the kinase activity, relieved by binding to $\mathrm{Ca}^{2+} /$ calmodulin. ${ }^{19}$ On the other hand, DAP-kinase catalytic activity was abolished by the substitution of a conserved lysine by alanine residue within the ATP-binding loop in the kinase domain (K42A), thus generating an inactive kinase with a potential dominantnegative activity (i.e., a loss of function mutation).
The biochemical studies of DAP-kinase were then complemented by functional assays. The cellular function was studied by transfection-based experiments. It was found that overexpression of wild-type DAP-kinase reduced the number of viable clones in HeLa cells. ${ }^{18}$ Detailed examination of the cells, early after the transfection (24$48 \mathrm{~h}$ ), indicated that overexpression of DAP-kinase imposed on the cells several morphological hallmarks of apoptosis. The constitutively active $\Delta \mathrm{CaM}$ mutant had stronger deathinducing effects in these overexpression assays. In contrast, the catalytically inactive kinase (K42A) completely lost its death inducing activity, and instead, its introduction slightly increased the number and the size of stable clones. The induction of cell death therefore strictly depended on the status of the intrinsic kinase activity, and the catalytic activity of this protein was clearly required for the deathpromoting function of this protein. The theme of cell death induction which is dependent on the catalytic activity of DAP-kinase repeated in a variety of tested cell lines such as 293 human embryonic kidney cells (Figure 2), REF52 fibroblasts, SV80 cells, and in primary embryonic fibroblasts. ${ }^{18,20,21}$ These overexpression experiments provided a second independent functional support for DAP-kinase being a pro-apoptotic gene, in addition to the initial finding that the anti-sense-RNA protected cells from apoptosis.

A

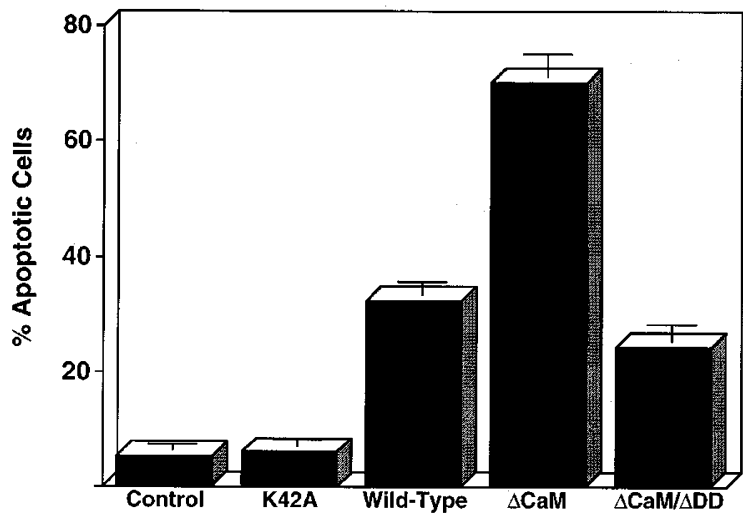

B

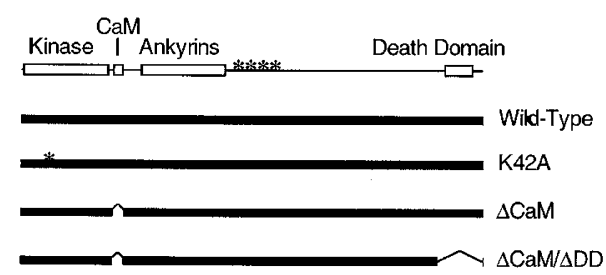

Figure 2 The apoptotic activity of DAP-kinase and its mutated derivatives. Trasient transfections of 293 human kidney cells with vectors coding for DAPkinase (either wild-type or the different loss of function and gain of function mutants). The constructs were co-transfected with the GFP vector to mark the transfected cells. Apoptotic cells were scored after $24 \mathrm{~h}$. (A) Apoptotic scores based on morphological changes among the GFP positive cells. (B) Schematic representation of DAP-kinase and of the mutants (the various deletions and the K42A point mutation) used in these experiments 
Finally it should be mentioned that it was found that the catalytically inactive mutant (K42A) of DAP-kinase functions in a trans-dominant negative manner. This possibility was examined by checking whether K42A may protect HeLa cells from IFN- $\gamma$ induced cell death. To this end, cells which were transfected with $\mathrm{K} 42 \mathrm{~A}$ or with an empty vector, as a control, were exposed to long term selections with IFN- $\gamma$. On average, the relative number of colonies that survived in the presence of IFN- $\gamma$ was fivefold higher in the K42A transfectants than in the corresponding vector-only transfectants. ${ }^{18}$ These results indicate that the K42A mutant can protect HeLa cells from IFN- $\gamma$-induced cell death, presumably by acting in a dominant negative manner, interfering with the normal function of the endogenous DAP-kinase. The dominant negative features provide a third evidence that DAP-kinase is a positive mediator of cell death, and that it mediates the apoptotic effects of IFN- $\gamma$.

\section{The death domain is important for the apoptotic function of DAP-kinase}

To gain insight into the death domain of DAP-kinase, a modelstructure which predicts the 3-dimensional organization of this region was constructed. The molecular modeling was based on the sequence similarity between DAP-kinase death domain and the closely related intracellular domain of the p75 neurotrophin receptor ${ }^{22}$ for which the NMR structure has been recently deposited in the Protein Data Bank. ${ }^{23}$ The predicted structure of DAP-kinase death domain consists of 99 amino acid residues that fold to form $6 \alpha$-helices. The model showed that similar to the death domain of p75 neurotrophin receptor, helices 1, 5 and 6 lie parallel to each other and are perpendicular to helices 2, 3 and $4 .{ }^{16}$ One notable difference between the two death domains is the presence of longer loops extending between helices 1 and 2 and 3 and 4 of DAPkinase death domain. It was postulated that since these loops are unique to DAP-kinase, they may contribute to the specificity of interaction with other proteins. ${ }^{16}$ Interestingly, a segment of the death domain spanning helices 2, 3 and 4 could efficiently compete, when ectopically expressed, with the full length protein, as assessed by inhibition of induction of apoptosis. Thus, a distinct substructure in this domain was defined.

To study the role of the death-domain in the apoptotic function of DAP-kinase, it was first tested whether its deletion may reduce the death-inducing functions of DAPkinase. This was initially assessed in transiently transfected 293 human embryonic kidney cells. ${ }^{20}$ The aforementioned constitutively active mutant of DAP-kinase $(\triangle \mathrm{CaM})$, which is a potent inducer of cell death, was employed in these experiments. Deletion of the death-domain from this mutant (creating the $\Delta \mathrm{CaM} / \Delta \mathrm{DD}$ mutant), significantly reduced the number of apoptotic cells ${ }^{20}$ (see also an example in Figure 2). It was therefore concluded that the death domain contributes to the death-inducing function of DAP-kinase and is a second important functional module in addition to the catalytic domain.

Since death domains of other known proteins were shown to mediate protein to protein interactions (either via homo- or heterodimerization), we postulated that the death domain of DAP-kinase, which contains all the functionally conserved regions, may also be involved in interactions with its specific partners, and thus may act in a dominantnegative manner. For that purpose, the death domain fragment (DD-DAPk) was subcloned into an expression vector. Co-transfection of the DD-DAPk with the $\triangle \mathrm{CaM}$ mutant of DAP-kinase reduced significantly cell death induced by DAP-kinase overexpression. ${ }^{20}$ These experiments demonstrated again the important role that the death domain plays in the death-promoting function of DAPkinase. Moreover, the demonstration that DD-DAPk can be used as a dominant negative fragment which blocks the action of the full length protein, provided a convenient tool to study the involvement of DAP-kinase in cell death induced by other external stimuli such as TNT- $\alpha$ or Fas.

Finally, it should be mentioned that no signs of homodimerization were detected when the death domain of DAP-kinase was tested by the yeast two-hybrid selection system (E Feinstein and A Kimchi, 2000, unpublished results). Also no indications for DAP-kinase dimerizations were obtained by co-immunoprecipitation experiments after ectopic expression of HA- and FLAG-tagged full length proteins in mammalian cell lines ( $T$ Spivak and A Kimchi, 2000, unpublished results). Thus, the major efforts are currently directed at identifying the relevant protein partner(s) whose specific interaction with this region may be essential for the death-promoting function of the kinase.

\section{Intracellular localization of DAP-kinase}

Immunostaining indicated that DAP-kinase is localized to the cytoskeleton in association with the microfilament system (Figure 1B). This was further supported by biochemical fractionation and by application of cytoskeletal disrupting drugs. ${ }^{18}$ Deletion analysis of DAP-kinase mapped the region mediating cytoskeleton binding to a stretch of 200 amino acids localized downstream to the first $\mathrm{P}$-loop motif ${ }^{18}$ (illustrated in Figure 1A). Most interestingly, cell killing by overexpression of DAP-kinase depended on its correct localization to the cytoskeleton. Expression of a truncated form of the kinase, catalytic active, yet mislocalized to the nucleus, failed to disrupt the actin microfilament and to kill the cells. ${ }^{18}$ This was an important finding since loss of stress fibers and disruption of microfilament organization occur at early stages of epithelial and fibroblastic cell death. The specific intracellular localization of the kinase may therefore provide a mechanistic clue as to how external signals impose these cytoskeletal changes.

Based on the modular structure of DAP-kinase comprising multiple protein-protein interacting domains, one could suggest a model in which DAP-kinase serves as a scaffold to bring together crucial elements of the death machinery. Multi protein complexes associated with the apoptotic process such as those formed proximal to the intracellular parts of the cytotoxic receptors or the complex that is formed at the vicinity of mitochondria (apoptosome) are well characterized. It will be of interest to find out whether DAPkinase could bring specific proteases or other kinases to the vicinity of their substrates in the cytoskeleton in addition to the direct phosphorylation of its putative substrates. 


\section{Expression pattern of DAP-kinase in normal tissues}

The tissue distribution of DAP-kinase was investigated in human, mice and rats. In mice, it was assessed by Northern blots and by in situ hybridization analyses. DAP-kinase mRNA was found to be ubiquitously expressed in various tissues. In brain and lung the levels of DAP-kinase mRNA were the highest, while moderate levels were found in kidney, heart and skin, and lower levels in stomach, spleen and thymus. ${ }^{24} \mathrm{~A}$ similar pattern of tissue distribution was found in human tissues (E Feinstein and A Kimchi, 2000, unpublished results). Developmental studies in mice showed that in the brain, DAPkinase mRNA appears at embryonic day 13 (E13) and was thereafter detected throughout the entire embryonic period. High levels of expression were detected in proliferative and postmitotic regions within cerebral cortex, hippocampus, and cerebellar Purkinje cells. ${ }^{24}$ These findings suggest that DAPkinase may play an important role in neurogenesis where a physiological type of cell death takes place. Interestingly, the overall expression of DAP-kinase mRNA in the brain gradually declined at postnatal stages, and the expression became restricted to hippocampus. ${ }^{24}$ Furthermore, it was found that the expression of DAP-kinase mRNA was increased prior to cell death induced by transient forebrain ischemia, indicating that in addition to the physiological type of cell death, DAP-kinase may be involved in neuronal cell death which occurs in response to external insults.

In rats, using in situ hybridization histochemistry, the expression of DAP kinase mRNA was observed in the mantle and ventricular zones of the entire neuraxis on embryonic day 15. Again, the overall expression in the brain decreased after birth and the expression was maintained at substantial levels in several restricted mature neuronal populations, such as olfactory bulb, hippocampal formation and cerebellar Purkinje and granule cells. ${ }^{25}$ In primary cultures prepared from hippocampal rat neurons the levels of DAP-kinase were very high, and efficient protection from cell death could be obtained by introducing into these neurons a peptide corresponding to the C-terminal tail of DAP-kinase ( $T$ Raveh and A Kimchi, 2000 , unpublished data). Obviously the ultimate function in various tissues, including the brain, will be assessed once DAP-kinase deficient mice have been generated.

\section{Involvement of DAP-kinase in various apoptotic pathways}

DAP-kinase was initially isolated by virtue of the ability of the corresponding anti-sense RNA fragment to protect HeLa cells from IFN- $\gamma$-induced apoptosis. An important question was whether this enzyme is part of a more general apoptotic machinery functioning downstream to various triggers or alternatively whether its function is limited to IFN signaling. The first hint of a broader involvement was provided once the original HeLa cells, stably transfected with anti sense DAPkinase cDNA fragment, were found to be protected from Fasinduced apoptosis as well. ${ }^{20}$ Once DAP-kinase mutants and fragments which function in a trans-dominant negative manner were identified, they provided additional tools to investigate the involvement of DAP-kinase in various apoptotic systems starting with the well studied responses to activated TNF- $\alpha$ and Fas receptors.

The examined systems consisted of 293 and HeLa cells which were transiently transfected with p55 TNF or Fas. In these experiments the ability of the death domain of DAPkinase (DD-DAPk) to protect from cell death induced by the ectopic expression of TNF- $\alpha$ or Fas receptors was tested. It was found that the co-expression of DD-DAPk significantly reduced the number of apoptotic cells. The DD-DAPk also protected from apoptosis induced by the ectopic expression of FADD/MORT1, an adapter that binds to the intracellular parts of Fas and TNF receptors via its specific death domain and is part of the death inducing signaling complex $(D I S C)^{20}$ (see also Figure 3A,B). These assays provided another line of evidence for DAP-kinase being a positive mediator in both TNF- $\alpha$ and Fas-induced cell death.

The protection conveyed by the death domain of DAPkinase was always partial (around $50-65 \%$ reduction in cell death). The effects of DD-DAPk were therefore clearly milder than the effects of the death domain of FADD/ MORT1 upon similar co-transfections (Figure $3 \mathrm{~A}$ ). This is not surprising considering the different functional position along the death pathways of the two proteins. FADD/ MORT- 1 acts in the proximity of Fas and TNF- $\alpha$ receptors and therefore its dominant negative mutant derivative blocks early receptor-generated events, such as the recruitment of the protease caspase- 8 to the receptor complex. As a consequence, it efficiently prevents most intracellular responses. DAP-kinase, in contrast, is not part of the DISC but rather functions further downstream. Beyond the receptor complex, the death pathways may diverge to several branches, and the partial protection conveyed either by anti-sense DAP-kinase RNA or by DDDAPk implies that DAP-kinase functions along some but not all these branches. Also the finding that DAP-kinasenegative cell lines, such as MCF7 or D122, can eventually be killed by TNF- $\alpha,{ }^{20,26}$ is consistent with the existence of DAP-kinase-dependent and independent branches within the network.

In a reciprocal experiment it was found that cell death induced by transfection with the activated mutant of DAPkinase $(\triangle \mathrm{CaM})$ failed to be rescued by the dominantnegative mutants of FADD/MORT1 and caspase-8 (Figure $3 \mathrm{C})$. These assays confirmed again that DAP-kinase functions downstream to the adapter protein FADD/ MORT1 and downstream to the protease caspase- $8 .^{20}$

Another link between DAP-kinase and TNF- $\alpha$ was found by a set of experiments that undertook an opposite approach. The gene was re-introduced by stable transfection into DAP-kinase null cells (D122 cells), and the transfected clone was assayed for its sensitivity to TNF- $\alpha$. It was found that restoration of DAP-kinase into cells that are DAP-kinase negative, accelerated TNF- $\alpha$-induced cell death. ${ }^{26}$ Another more interesting observation was that reintroduction of normal levels of DAP-kinase into the D122 cells restored their sensitivity to apoptotic cell death induced by anchorage-independent growth conditions. ${ }^{26}$ The latter further extended the spectrum of apoptotic signals that impinge upon DAP-kinase. 


\section{Involvement of DAPk in TNF/Fas-induced apoptosis}

A

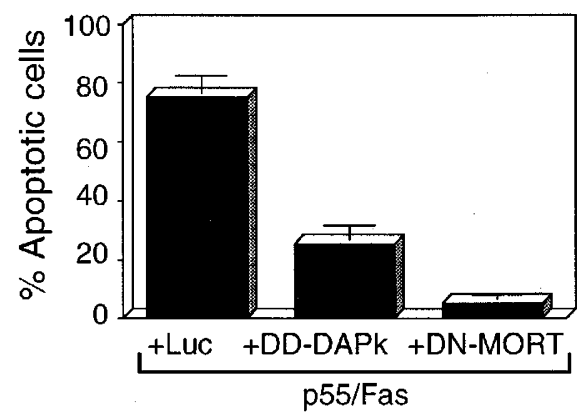

B

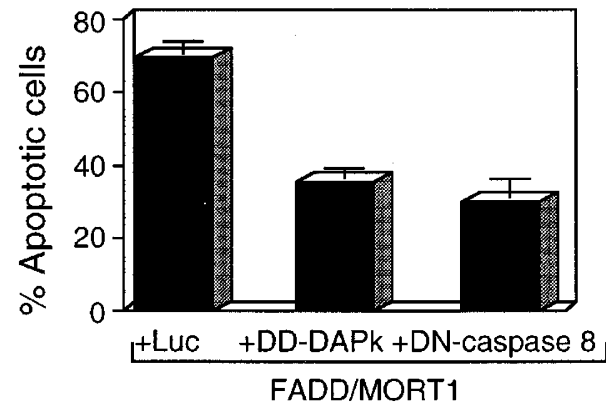

C

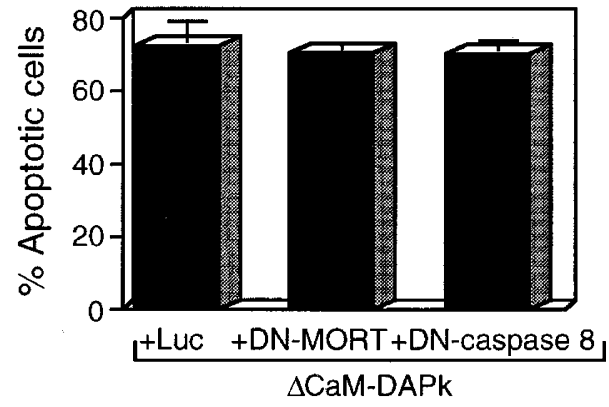

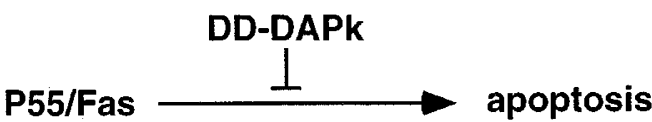

FADD/MORT1

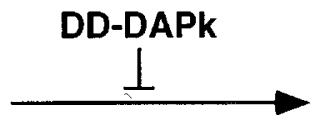

apoptosis

Figure 3 Involvement of DAP-kinase in TNF/Fas cell death pathways. Transient transfections of 293 cells with vectors encoding death inducers genes - p55TNFR or Fas, FADD/MORT1 or $\triangle \mathrm{CaM-DAP-kinase} \mathrm{together} \mathrm{with} \mathrm{vectors} \mathrm{encoding} \mathrm{the} \mathrm{indicated} \mathrm{proteins} \mathrm{tested} \mathrm{for} \mathrm{possible} \mathrm{protection.} \mathrm{A} \mathrm{summary} \mathrm{of} \mathrm{the} \mathrm{results} \mathrm{is}$ depicted in a schematic fashion, for each set of experiments, at the right part of the Figure

\section{DAP-kinase as a tumor suppressor gene}

Since selection against positive mediators of apoptosis is an advantageous step in cancer development, it became of interest to look at the status of DAP genes in the multi-step tumorigenesis process. The first hint for involvement of DAPkinase in the tumorigenesis process emerged from the notion that DAP-kinase expression was lost in various tumor cell lines. ${ }^{27}$ It was found in this respect that DAP-kinase mRNA and protein expression levels were below detection limits in $80 \%$ of B-cell lymphoma and leukemia cell lines, and in 30$40 \%$ of cell lines derived from bladder carcinomas, breast carcinomas, and renal cell carcinomas. This stood in contrast to the finding that DAP-kinase mRNA is ubiquitously expressed in immortalized cell lines established from non tumorigenic cell sources ${ }^{27}$ and in normal tissues and primary cell cultures. $^{23,24}$

Subsequent attempts were devoted at elucidating the mechanisms responsible for this loss of expression. Gene silencing by methylation is well documented and has been demonstrated for various tumor suppressor genes, such as $\mathrm{p} 16, \mathrm{VHL}$ and $\mathrm{pRB}$ during tumorigenesis (reviewed $\mathrm{in}^{28}$ ). To test whether DNA methylation might have a role in silencing of DAP-kinase expression in some of the cancer cell lines, experiments utilizing a demethylating drug 5'-aza-2'deoxycytidine were performed. In cell lines derived from two cases of bladder carcinoma and one case of B-cell lymphoma in which DAP-kinase was not expressed, treatment of cells with the 5'-aza-2'-deoxycytidine restored the expression of DAP-kinase to these cell lines. However, 
in some other cases, this treatment of cell lines did not restore DAP-kinase expression, indicating that DNA methylation is one out of several mechanisms involved in shutting off DAP-kinase expression. ${ }^{27}$ Interestingly, in Raji, a fully methylated, DAP-kinase nonexpressing Burkitt's lymphoma cell line, the demethylation by 5 -aza-2'-deoxycytidine treatment restored their apoptotic responses to IFN $-\gamma,{ }^{30}$ thus providing a physiological relevance to this loss of expression.

Recent work by the laboratory of JG Herman ${ }^{29,30}$ tested the methylation status of the DAP-kinase gene in fresh tumor specimens from patients with small cell lung cancer and primary B-cell malignancies. For this screen the Methylation Specific PCR (MSP) technique which was previously developed by Herman et $a /,{ }^{31}$ was used. This PCR method detects methylations of $\mathrm{CpG}$ sites in $\mathrm{CpG}$ islands. It is based on chemical modification of cytosine to uracil by sodium bisulfide treatment. In these reactions all cytosines are converted to uracils, but those that are methylated are resistant to this modification and remain as cytosine. Primers specific for either methylated or the modified unmethylated DNA are then used for DNA amplifications. Methylation in CpG sites is scored by gel fractionations of the amplified DNA and detecting positive signals with the corresponding primers. The primer sequences for DAP-kinase were derived from the $5^{\prime}$ region of the gene which contains a typical GpC island. All the normal tissues had unmethylated copies exclusively. In contrast, $23 \%$ of the tested small cell lung tumors, $84 \%$ of the B cell lymphoma and $100 \%$ of the Burkitt's lymphomas displayed DAP-kinase methylations. ${ }^{29,30}$ Using the same technique, we found in our laboratory that $26 \%$ of tested breast and colon carcinoma cancers displayed methylation of DAP-kinase CpG island ( $\mathrm{J}$ Kissil and A Kimchi, 2000, unpublished data). Additionally, it has been recently reported, that DAP-kinase was hypermethylated in $18 \%$ of primary tumors from head and neck cancer patients. Most importantly, a statistically significant correlation was obtained between the presence of promoter methylation and lymph node involvement and advanced stage disease. $^{32}$ Thus, the DAP-kinase gene is abnormally methylated in a significant portion of human tumors, providing the first proof that inactivation of this gene is critical for the development of these common malignancies. Yet, as is the case for the other tumor suppressor genes, and as predicted from the 5'-aza-2'-deoxycytidine experiments discussed above, DNA methylation is not an exclusive way to inactivate DAP-kinase. Loss or inactivation may also result from mutations, deletions, or other DNA rearrangements; two $\mathrm{LOH}$ cases were already identified in our initial screen of breast and colon carcinoma cancers ( $\mathrm{J}$ Kissil and A Kimchi, 2000, unpublished data). A summary of the status of DAP-kinase in human tumors and cancer cell lines is shown in Table 1.

An animal model system which was employed in our laboratory to test the involvement of DAP-kinase in tumorigenesis, was based on the finding that highly metastatic clones originating from a Lewis lung cell carcinoma cells were DAP-kinase negative, whereas the low metastatic counterparts of these lung carcinoma cells expressed normal DAP-kinase levels. ${ }^{26}$ Subsequently, DAP-kinase expression was restored by transfection in the highly metastatic clones, yielding clones expressing the transgene at physiological levels, with no changes in the overall pattern of their growth in vitro. The metastatic activity of these genetically manipulated cells was examined by intravenous injection of the cells into syngeneic mice. Strikingly, restoration of physiological levels of DAP-kinase into the highly metastatic Lewis carcinoma cells suppressed their ability to form lung metastases in mice. ${ }^{26}$ Conversely, rare lung lesions which were spontaneously selected in mice following injection of the original poorly metastatic cells, lost endogenous DAPkinase expression at high frequency. Altogether, these experiments suggested that loss of DAP-kinase expression provides a positive selective advantage during the formation of lung metastases. The transgene also delayed local tumor growth, yet with lower efficiency than the suppression of the metastatic activity.

The mechanisms underlying the suppressive effects of DAP-kinase on metastasis and local tumor growth were then studied. In situ TUNEL staining was performed on histological sections of local tumors. It was found that the apoptotic index in the slow growing local tumors, formed by the DAP-kinase-transfected cells, was higher than the value measured in the tumor mass formed by the control clone. These results indicated that expression of the DAP-kinase transgene re-sensitized the tumor cells to apoptotic stimuli, a property which they had originally managed to circumvent by losing DAP-kinase. To directly address this point, the

Table 1. The status of DAP-kinase in human tumors and cancer human cell lines

\begin{tabular}{lcr}
\hline Type of tumor (\%) & Aberration & Reference \\
\hline B cell lymphoma (84) & DNA methylation & 30 \\
Non small lung cancer (23) & DNA methylation & 29 \\
Head and neck cancer (18) & DNA methylation & 32 \\
Colon carcimoma (26) & DNA methylation & JL Kissil and A Kimchi, 2000 (unpublished) \\
Colon and breast cancers (15) & JL Kissil and A Kimchi, 2000 (unpublished) \\
& \\
\hline Tumor cell lines with no DAPk expression (\%) & \\
B cell leukemia (80) & \\
Breast carcinoma (30) & \\
Bladder carcinoma (29) & \\
Renal carcinoma (40) & \\
\hline
\end{tabular}


transfected cells were exposed in vitro to different apoptotic stimuli, and found to be extremely sensitive to TNF- $\alpha$ or to detachment from extracellular matrix. ${ }^{26}$ Based on these experiments, it has been suggested that DAP-kinasemediated suppression of metastasis results, at least in part, from increased sensitivity to various death-inducing stimuli. These experiments propose that loss of DAP-kinase expression provides a novel mechanism that links suppression of apoptosis to metastasis.

\section{The novel family of DAP-kinase related proteins}

Several years after the identification of DAP-kinase, a few novel protein kinase members were identified and cloned, each displaying strong homology to DAP-kinase in their catalytic domains (Figure 4). Based on these criteria they were classified altogether as members of a novel subfamily of kinases - the DAP-kinase related proteins. The most homologous kinase to DAP-kinase is DRP-1 (DAP-kinase Related Protein-1 also named DAPK-2), ${ }^{33,34}$ a 42 kDa protein which shows high degree of homology $(80 \%$ identity at the amino-acid level) to DAP-kinase both in its catalytic domain and its calmodulin-regulatory region, but not to other domains of DAP-kinase. Consistent with the structural homology within the kinase domain, DRP-1 was found to phosphorylate MLC, like DAP-kinase, in a $\mathrm{Ca}^{2+} / \mathrm{CaM}$-dependent manner. Yet, the divergence from DAP-kinase in the extra-catalytic domains imposes some critical features which are unique to DRP-1. This kinase was found to be localized in the cytoplasm as a soluble protein, which tends to homodimerize via its carboxy terminal tail. ${ }^{33}$

Most importantly, DRP-1 promotes cell death when it is ectopically expressed in primary cell cultures and immortalized cell lines. As in the case of DAP-kinase, the apoptotic effects of DRP-1 strictly depend on the status of its catalytic activity. Additionally, the C-terminal tail is also required for the apoptotic functions, yet it is necessary as long as the CaM-regulatory domain is present, suggesting that it may regulate the CaM responses. ${ }^{33}$ Since the death promoting effects of DRP-1 could be prevented by a dominant negative fragment of DAP-kinase, a functional interaction between the two kinases has been proposed placing DRP1 upstream to DAP-kinase. ${ }^{33}$

A second protein, ZIP-kinase (also named Dlk), was also found to be about $80 \%$ identical to DAP-kinase again only within the kinase domain. ${ }^{35,36}$ Unlike DRP-1, ZIPkinase lacks the CaM-regulatory domain and is localized to the nucleus. It interacts with the ATF-4 transcription factor. The activation of ZIP kinase occurs by a mechanism involving homodimerization, mediated by its C-terminal leucine zipper domain. Overexpression of intact ZIP-kinase, but not of catalytically inactive kinase mutants, led to morphological changes characteristic of apoptosis. ${ }^{35}$ In some cell systems the transient expression of the rat ZIP-kinase did not result in any signs of apoptosis. ${ }^{36}$ Yet, an interesting twist was recently reported in these systems, according to which the interaction with Par-4, a modulator of the transcription factor WT1, changed the intracellular localization of DIk/ZIP to the cytoplasm, particularly to actin filaments. This further provoked a reorganization of the cytoskeleton and morphological symptoms of apoptosis. ${ }^{37}$

Two other novel proteins, DRAK1 and DRAK2, closely related to each other, and sharing about $50 \%$ identity with the kinase domain of DAP-kinase, were also recently characterized. $^{38}$ Like ZIP-kinase, DRAK1 and DRAK2 also lack the CaM-regulatory domain. Overexpression of these proteins induced apoptosis, dependent on the functionality of their kinase domain. Thus, it appears that DRP-1, ZIPkinase and DRAK1/2 which are closely related to DAPkinase may also possess a unique functional role in mediation of apoptosis which seems to rely on their kinase activity. The high similarity of these proteins in their kinase domain might indicate common substrates or that these kinases may function along a common pathway by activating each other. These possibilities are currently under intensive investigation.

\section{The DAP-kinase family of proteins}

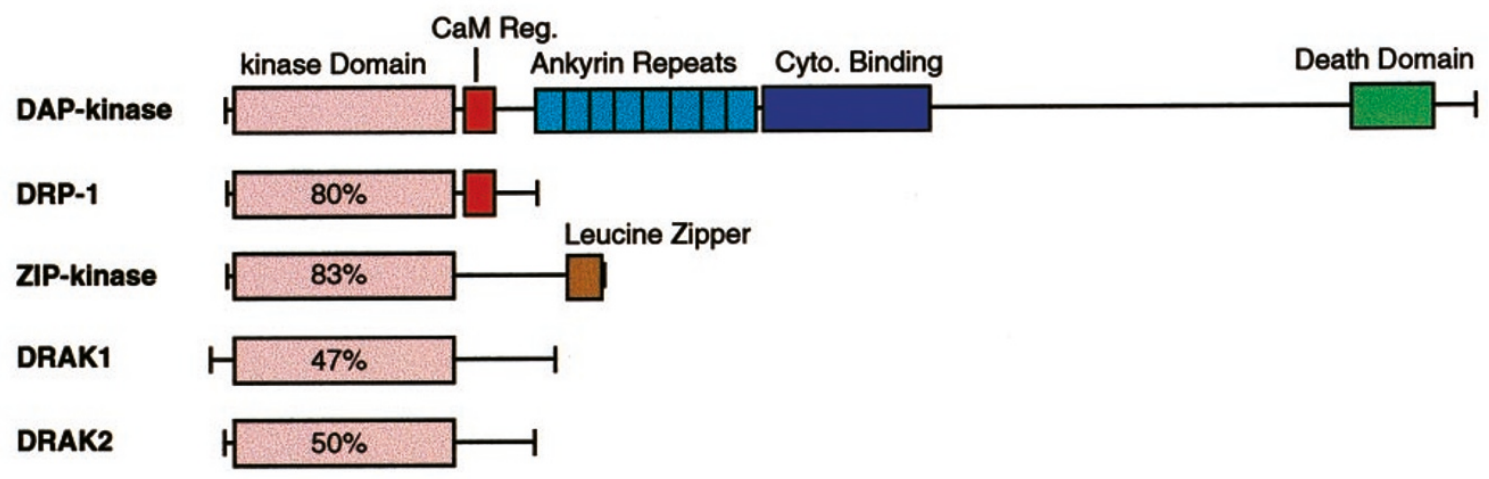

Figure 4 The DAP-kinase family of proteins. The following structural domains are boxed: catalytic domain (pink), CaM regulatory region (red), ankyrin repeats (blue), cytoskeleton-binding domain (cyan), death domain (green) and leucine zipper motif (brown). Numbers shown in the kinase boxes correspond to percentage of identity at the amino acid level to the catalytic domain of DAP-kinase 


\section{Conclusions and prospects for the future}

DAP-kinase was initially identified as a gene whose antisense-mediated reduced expression protected HeLa cells from IFN- $\gamma$ induced cell death. ${ }^{12}$ This was the first indication that linked the function of this gene to programmed cell death. The work in the last few years has established the role of DAP-kinase as a general mediator of cell death common to several apoptotic triggers. DAP-kinase was shown to be involved in apoptosis initiated by several cytokines (e.g. IFN- $\gamma$, TNF- $\alpha$, Fas), ${ }^{20}$ by detachment from extracellular matrix ${ }^{26}$ and by oncogenes. ${ }^{21}$ Moreover, DAP-kinase was found, by several criteria to be involved in the multi-step process of tumorigenesis. ${ }^{21,26,27,30,32}$ The role of DAP-kinase in cancer was found to deviate from its role in cell death. This wide involvement in apoptosis approves the basic assumptions of the TKO system as well as the importance of the biochemical and functional characterization of DAP-kinase.

While the characterization of DAP-kinase is still very far from completion, the first steps in understanding the function of DAP-kinase have been achieved in the past few years. The multi-domain structure of DAP-kinase has opened the detailed examination of each domain and the study of their contribution to the function of DAP-kinase. Three domains of DAP-kinase were found to be important for its function in cell death: (1) the kinase domain and the CaM binding domain which controls the catalytic activity; (2) the cytoskeleton binding domain responsible for the correct localization of the kinase and (3) the death domain that probably mediates interaction with some other proteins. The importance of these domains might direct the future work in several directions. First of all the identity of the direct substrates for DAP-kinase should be revealed. Once these substrates have been identified, then the detailed mechanisms coupling the kinase to downstream targets may be deciphered. Myosin light chain (MLC) is one of the substrates that is efficiently phosphorylated in vitro by DAPkinase. Recently, MLC phosphorylation was linked to membranal blebbing. ${ }^{39}$ Whether this reflects one of the functional arms of DAP-kinase is an open question to be further investigated in the future. Second, the significance of the cytoskeletal localization of DAP-kinase is still not fully clear. Does the cytoskeleton serve only to recruit several proteins, including DAP-kinase, to form an apoptotic complex, or does DAP-kinase directly regulate cytoskeletal components by phosphorylating them? Third, the proteins that bind to the death domain of DAP-kinase must be identified. These proteins should be critical for the apoptotic activity of DAP-kinase and it is hard to speculate at the present time how they will function and affect DAP-kinase.

Many questions regarding the regulation of DAP-kinase are still open. DAP-kinase was shown to be regulated by $\mathrm{Ca}^{2+}$ and CaM. This means that the calcium spike arising in apoptosis is one of the participants that activate the kinase. Yet additional layers of regulation might exist which should restrain, on the one hand, the pro-apoptotic functions of the kinase in healthy cells, and might co-operate and/or prolong the calcium signals, on the other hand. DAP-kinase was shown to undergo autophosphorylation whose functional implication is currently being investigated. DAP-kinase might also be phosphorylated by other kinases, for example by the newly identified DAP-kinase-related kinases. Changes in subcellular localization or interaction with other protein partners might be another way to regulate DAP-kinase.

Altogether, the broad involvement of DAP-kinase in apoptosis and cancer, the complex structure of the protein, the many known and yet unknown aspects of its function, promise that DAP-kinase will be in the focus of many studies in the fields of apoptosis and tumorigenesis.

\section{References}

1. Hengartner MO (1996) Programmed cell death in invertebrates. Curr. Opin. Genet. Dev. 6: $34-38$

2. Hengartner MO (1995) Out-of body experiences: cell-free cell death. Bioessays 17: $549-552$

3. Wallach D, Boldin MP, Kovalenko AV, Malinin NL, Mett IL and Camonis JH(1998) The yeast two-hybrid screening technique and its use in the study of proteinprotein interactions in apoptosis. Curr. Opin. Immunol. 10: 131-136

4. Kimchi A (1998) DAP genes: novel apoptotic genes isolated by a functional approach to gene cloning. Biochim. Biophys. Acta. 1377: F13-F33

5. Deiss LP and Kimchi A (1991) A genetic tool used to identify thioredoxin as a mediator of a growth inhibitory signal. Science 252: 117-120

6. Levy-Strumpf $\mathrm{N}$ and Kimchi A (1998) Death associated proteins (DAPs): from gene identification to the analysis of their apoptotic and tumor suppressive functions. Oncogene 17: $3331-3340$

7. Levy-Strumpf N, Deiss LP, Berissi H and Kimchi A (1997) DAP-5, a novel homolog of eukaryotic translation initiation factor $4 G$ isolated as a putative modulator of gamma interferon-induced programmed cell death. Mol. Cell. Biol. 17: $1615-1625$

8. Henis-Korenblit S, Strumpf NL, Goldstaub D and Kimchi A (2000) A novel form of DAP-5 protein accumulates in apoptotic cells as a result of caspase cleavage and internal ribosomal entry site - mediated translation. Mol. Cell. Biol., 20: 496506

9. Kissil JL, Deiss LP, Bayewitch M, Raveh T, Khaspekov G and Kimchi A (1995) Isolation of DAP-3: a novel mediator of interferon- $\gamma$-induced cell death. J. Biol. Chem. 270: 27932-27936

10. Kissil JL, Cohen O, Raveh T and Kimchi A (1999) Structure-function analysis of an evolutionary conserved protein, DAP3, which mediates TNF-alpha- and fasinduced cell death. EMBO J. 18: 353-362

11. Deiss LP, Galinka H, Berissi H, Cohen O and Kimchi A (1996) Cathepsin D protease mediates programmed cell death induced by interferon-gamma, Fas/ APO-1 and TNF-alpha. EMBO J. 15: 3861-3870

12. Deiss LP, Feinstein E, Berissi H, Cohen O and Kimchi A (1995) Identification of a novel serine/threonine kinase and a novel $15-\mathrm{kD}$ protein as potential mediators of the gamma interferon-induced cell death. Genes Dev. 9: 15-30

13. Feinstein E, Kimchi A, Wallach D, Boldin M and Varfolomeev E (1995) The death domain: a module shared by proteins with diverse cellular functions. Trends Biochem. Sci. 20: 342-344

14. Sedgwick SG and Smerdon SJ (1999) The ankyrin repeat: a diversity of interactions on a common structural framework. Trends Biochem. Sci. 24:311316

15. Baker SJ and Reddy EP (1998) Modulation of life and death by the TNF receptor superfamily. Oncogene 17: $3261-3270$

16. Raveh T, Berissi H, Eisenstein M, Spivak T and Kimchi A (2000) A functional genetic screen identifies regions at the $c$-terminal tail and death domain of DAPkinase, that are critical for its proapoptotic activity. Proc. Natl. Acad. Sci. USA 97: $1572-1577$

17. Itoh $\mathrm{N}$ and Nagata $\mathrm{S}$ (1993) A novel protein domain required for apoptosis. Mutational analysis of human Fas antigen. J. Biol. Chem. 268: 10932-10937

18. Cohen O, Feinstein E and Kimchi A (1997) DAP-kinase is a Ca2+/calmodulindependent, cytoskeletal-associated protein kinase, with cell death-inducing functions that depend on its catalytic activity. EMBO J. 16: 998-1008 
19. Shoemaker MO, Lau W, Shattuck RL, Kwiatkowski AP, Matrisian PE, GuerraSantos L, Wilson E, Lukas TJ, Van Eldik LJ and Watterson DM(1990) Use of DNA sequence and mutant analyses and antisense oligodeoxynucleotides to examine the molecular basis of nonmuscle myosin light chain kinase autoinhibition, calmodulin recognition, and activity. J. Cell. Biol. 111: 11071125

20. Cohen O, Inbal B, Kissil JL, Spivak-Kroizman T, Raveh T, Berissi H, Feinstein E and Kimchi A (1999) DAP-kinase participates in TNF- $\alpha$ and Fas-induced apoptosis and its function requires the death domain. J. Cell. Biology 146: 141 148

21. Raveh T, Droguett G, Cohen O, Horwitz MS, DePinho RA and Kimchi A (2000) DAP-kinase Activates a p19ARF/p53-mediated Apoptoltic Checkpoint to Suppress Oncogenic Transformation. Nature Cell. Biol. (in press)

22. Liepinsh E, llag LL, Otting G and lbanez CF (1997) NMR structure of the death domain of the p75 neurotrophin receptor. EMBO J. 16: 4999-5005

23. Bernstein FC, Koetzle TF, Williams GJ, Meyer EF, Brice MD, Rodgers JR, Kennard O, Shimanouchi T and Tasumi M (1977) The Protein Data Bank. A computer-based archival file formacromolecular structures. Eur. J. Biochem. 80: $319-324$

24. Yamamoto M, Takahashi H, Nakamura T, Hioki T, Nagayama S, Ooashi N, Sun X, Ishii T, Kudo Y, Nakajima-lijima S, Kimchi A and Uchino S (1999) Developmental changes in distribution of death-associated protein kinase mRNAs. J. Neurosci. Res. 58: 674-683

25. Sakagami $H$ and Kondo $H$ (1997) Molecular cloning and developmental expression of a rat homologue of death-associated protein kinase in the nervous system. Brain Res. Mol. Brain Res. 52: 249-256

26. Inbal B, Cohen O, Polak-Charcon S, Kopolovic J, Vadai E, Eisenbach L and Kimchi A (1997) DAP kinase links the control of apoptosis to metastasis. Nature 390: $180-184$

27. Kissil J, Feinstein E, Cohen O, Jones PA, Tsai YC, Knowles MA, Eydmann ME and Kimchi A (1997) DAP-kinase loss of expression in various carcinoma and Bcell lymphoma cell lines: possible implications for role as tumor suppressor gene. Oncogene 15: 403-407

28. Laird PW (1997) Oncogenic mechanisms mediated by DNA methylation. Molecular Medicine Today 3: 223-229

29. Esteller M, Sanchez-Cespedes M, Rosell R, Sidransky D, Baylin SB and Herman JG (1999) Detection of aberrant promoter hypermethylation of tumor suppressor genes in serum DNA from non-small cell lung cancer patients. Cancer Res. 59: $67-70$
30. Katzenellenbogen RA, Baylin SB and Herman JG (1999) Hypermethylation of the DAP-Kinase CpG island is a common alteration in B-cell malignancies. Blood 93: $4347-4353$

31. Herman JG, Graff JR, Myohanen S, Nelkin BD and Baylin SB (1996) Methylationspecific PCR: a novel PCR assay for methylation status of $\mathrm{CpG}$ islands. Proc. Natl. Acad. Sci. USA 93: $9821-9826$

32. Sanchez-Cespedes M, Esteller M, Wu L, Nawroz-Danish H, Yoo GH, Koch WM Jen J, Herman JG and Sidransky D (2000) Gene promoter hypermethylation in tumors and serum of head and neck cancer patients. Cancer Res. 60: 892-895

33. Inbal B, Shani G, Cohen O, Kissil JL and Kimchi A (2000) Death - associated protein kinase - related protein 1, a novel serine/threonine kinase involved in apoptosis. Mol. Cell. Biol. 20: 1044-1054

34. Kawai T, Nomura F, Hoshino K, Copeland NG, GilbertDJ, Jenkins NA and Akira S (1999) Death-associated protein kinase 2 is a new calcium/calmodulindependent protein kinase that signals apoptosis through its catalytic activity. Oncogene 18: $3471-3480$

35. Kawai T, Matsumoto M, Takeda K, Sanjo Hand Akira S (1998) ZIP kinase, a novel serine/threonine kinase which mediates apoptosis. Mol. Cell. Biol. 18: 1642 1651

36. Kogel D, Plottner O, Landsberg G, Christian S and Scheidtmann KH (1998) Cloning and characterization of Dlk, a novel serine/threonine kinase that is tightly associated with chromatin and phosphorylates core histones. Oncogene 17: 2645-2654

37. Page G, Kogel D, Rangnekar V and Scheidtmann KH (1999) Interaction partners of DIk/ZIP kinase: co-expression of DIk/ZIP kinase and Par-4 results in cytoplasmic retention and apoptosis. Oncogene 18: 7265-7273

38. Sanjo H, Kawai T and Akira S (1998) DRAKs, novel serine/threonine kinases related to death-associated protein kinase that trigger apoptosis. J. Biol. Chem. 273: $29066-29071$

39. Mills JC, Stone NL, Erhardt J and Pittman RN (1998) Apoptotic membrane blebbing is regulated by myosin light chain phosphorylation. J. Cell. Biol. 140: $627-636$ 